Actes du XXVe Congrès International de Linguistique et

de Philologie Romanes

Innsbruck, 3 - 8 septembre 2007 Tome I - VII

Hrsg. v. Iliescu, Maria / Siller-Runggaldier, Heidi M. /

Danler, Paul

Tome I-VII

August 2010. 24 x 17 cm. LII, 5027 pages. Relié. Euro [D] 989,- / für USA, Kanada, Mexiko US\$ 1.533,-.

ISBN 978-3-11-023191-5

Tome I-VII

Ed. par Iliescu, Maria / Danler, Paul / Siller, Heidi M.

August 2010. eBook (derzeit nur für Bibliotheken / Institutionen). Unverb.

Ladenpreis Euro [D] 989,- / für USA, Kanada, Mexiko US\$ 1.533,-.

ISBN 978-3-11-023192-2

Tome I

August 2010. 24 x 17 cm. XII, 718 pages. Relié. Euro [D] 164,95 / für USA, Kanada, Mexiko US\$256,-.

ISBN 978-3-11-023193-9

Tome II

August 2010. 24 x 17 cm. XIV, 911 pages. Relié. Euro [D] 164,95 / für USA, Kanada, Mexiko US\$256,-.

ISBN 978-3-11-023195-3

Tome III

August 2010. 24 x 17 cm. XIV, 797 pages. Relié. Euro [D] 164,95 / für USA, Kanada, Mexiko US\$256,-.

ISBN 978-3-11-023197-7

Tome IV

August 2010. 24 x 17 cm. XII, 627 pages. Relié. Euro [D] 124,95 / für USA, Kanada, Mexiko US\$194,-.

ISBN 978-3-11-023199-1

Tome $\mathrm{V}$

August 2010. 24 x 17 cm. XII, 629 pages. Relié. Euro [D] 124,95 / für USA, Kanada, Mexiko US\$ 194,--

ISBN 978-3-11-023201-1

Tome VI

August 2010. 24 x 17 cm. XII, 661 pages. Relié. Euro [D] 124,95 / für USA, Kanada, Mexiko US\$ 194,--

ISBN 978-3-11-023203-5

Tome VII

August 2010. 24 x 17 cm. XII, 633 pages. Relié. Euro [D] 124,95 / für USA, Kanada, Mexiko US\$ 194,-.

ISBN 978-3-11-023205-9 
Actes du $\mathrm{XXV}^{\mathrm{e}}$ Congrès International de Linguistique et de Philologie Romanes

TOME III 


\section{$\mathrm{XXV}^{\mathrm{e}}$ CILPR \\ Congrès International \\ de Linguistique \\ et de Philologie Romanes \\ 3-8 septembre 2007 \\ Innsbruck}

De Gruyter 


\title{
Actes du
}

\section{$\mathrm{XXV}^{\mathrm{e}}$ Congrès International}

de Linguistique

et de Philologie Romanes

\author{
Innsbruck 2007
}

Éditeurs:

Maria ILIESCU, Heidi SILLER-RUNGGALDIER, Paul DANLER

\section{TOME III}

Section 7: Sémantique paradigmatique, syntagmatique et cognitive; phraséologie / collocations

Section 8: Onomastique (toponymie et anthroponymie)

Section 9: Constitution de la norme dans les langues romanes

\section{De Gruyter}


ISBN 978-3-11-023197-7 (Tome III)

Gesamt-ISBN 978-3-11-023191-5 (Tome I-VII)

e-ISBN 978-3-11-023192-2 (Tome I-VII)

\section{Bibliografische Information der Deutschen Nationalbibliothek}

Die Deutsche Nationalbibliothek verzeichnet diese Publikation in der Deutschen Nationalbibliografie; detaillierte bibliografische Daten sind im Internet über http://dnb.d-nb.de abrufbar.

(C) 2010 Walter de Gruyter GmbH \& Co. KG, Berlin/New York

Satz: Georg Rosensteiner

Druck und Einband: Hubert \& Co. GmbH \& Co. KG, Göttingen

$\infty$ Gedruckt auf säurefreiem Papier

Printed in Germany

www.degruyter.com 


\section{Matteo Milani}

\section{Panorama delle prime grammatiche tra latino e volgare italiano}

I testi grammaticali redatti e trascritti nella penisola italiana tra la fine del Duecento e il pieno Quattrocento tracciano complessivamente un percorso di cambiamento lento, ma continuo, che, attraverso una sempre più profonda revisione dei modelli classici, porterà infine alla codificazione e alla diffusione degli strumenti didattici ormai pienamente rinascimentali. ${ }^{1}$

In tale processo gioca un ruolo fondamentale l'apertura progressiva ai nuovi codici linguistici volgari, inizialmente applicati ai livelli più avanzati del curriculum scolastico per la glossatura degli autori classici e per gli esercizi di traduzione (themata), estesi poi alle prime fasi dell'insegnamento di livello elementare come strumento di accesso alla lingua latina. $^{2}$

Quest'ultima tendenza trova riscontro, più che nella presenza di sporadiche citazioni volgari all'interno di trattati ancora tutti latini, nella stesura dei primi glossari e delle prime grammatiche latino-volgari, collocabili tra i secoli XIV e XV, ma con anticipazioni già alla fine del XIII. ${ }^{3}$

Tralasciando i primi ${ }^{4}$, entro i limiti del presente studio cercheremo di orientarci nel variegato panorama delle grammatiche latino-volgari italiane tardo-medievali: un'operazione, diremo subito, non semplice, a causa dell'accentuata frammentazione linguistica e della disarticolata distribuzione cronologica e geografica dei testi in esame, per di più privi in qualche caso di un'edizione (critica) moderna sulla quale fare sicuro affidamento.

Con l'obiettivo ultimo di individuare, attraverso un'indagine comparativa, alcuni sentieri intertestuali atti a collegare $\mathrm{i}$ singoli rappresentanti di questa particolare tipologia di

1 Sulla fase conclusiva di questo percorso si veda in primo luogo Rizzo 1996.

2 Sintetico quadro riassuntivo in Casapullo 1999: 93-101. Per l'area veneta, si vedano in particolare i riferimenti contenuti in Belloni / Pozza 1987: 28 n. 5, cui aggiungere Nardi 1971.

3 Sul confine tra le grammatiche propriamente latine e i nuovi trattati latino-volgari possiamo porre, tra gli altri, il Compendium Grammaticae di Pietro da Isolella, pubblicato in Fierville 1886, e la Grammatica et orthographia di Folchino dei Borfoni, oggi inclusa in De Santis 2003. Sul lato opposto, paiono per esempio già orientate verso le nuove soluzioni umanistiche le Regulae grammaticales di Zomino da Pistoia.

4 Principali riferimenti in Baldelli 1959, cui aggiungere almeno D'Agostino 1983, Gualdo 1999 e Sabatini 1963-1964. 
produzione didattica, vale forse la pena partire proprio da una mappatura delle opere fino ad oggi pubblicate (talvolta solo parzialmente) o almeno commentate: ${ }^{5}$

Italia settentrionale: Frammenti grammaticali di Biella, XIII-XIV ( $F B i$; Gasca Queirazza 1966); Frammento grammaticale comense, XVex ( $F C o$; Milani 2005); Frammento di grammatica latino-bergamasca, XIII-XIV ( $F B g$; Sabbadini 1904); Reliquie volgari di scuola bergamasca, XIVin (RBg; Contini 1934); Grammatica latino-veronese, XIII-XIV (GVr; De Stefano 1905); Grammatica latino-veneta (padovana), XIII-XIV (GPd; Manacorda 1913-1914); Guarino Guarini, Regulae grammaticales, Venezia (o Verona) ante 1418 (GG; cf. infra); Maffeo Valaresso, Regule, Venezia 1432 (RVe; Segarizzi 19151916); Frammenti grammaticali latino-friluani, Cividale del Friuli XIVex ( $F F$; Schiaffini 1921); Esercizi di versione dal volgare friulano, Cividale del Friuli XIVex ( $E F$; Schiaffini 1922); Frammenti grammaticali latino-volgari dell'Archivio di Stato di Cuneo, XIII-XIV (FCn; Piccat 1988);

Italia centrale: Francesco da Buti, Regulae grammaticales, Pisa 1355-1378 (FdB; Martinelli); Goro d'Arezzo, Regulae parvae, Arezzo XIV (GdA; Marchesi 1910); Cristiano da Camerino, Regule, Italia mediana (Perugia?) XIVex (CdC; Lovito 1994-1995);

Italia meridionale: Nicola de Aymo, Interrogatorium constructionum gramaticalium, Lecce 1444 (NdA; Coluccia / Greco / Scarpino 2005); Nicola de Aymo, Interrogatorium constructionum gramaticalium, Puglia 1454 (NdAN; Coluccia / Greco / Scarpino 2005: $134-141)^{7}$

Non vengono inclusi in tale rassegna gli esercizi di traduzione (themata) redatti e/o tramandati in forma isolata, ovvero non inseriti all'interno di una trattazione grammaticale o comunque non accompagnati da questa: è il caso degli esercizi genovesi trascritti da Alfredo Schiaffini (1922: 88-89 n. 4), di quelli trevigiani segnalati da Alfredo Stussi (1968), di quelli veneti pubblicati da Gino Belloni e Marco Pozza (1987: 27-44) ${ }^{8}$, di quelli del fiorentino Bartolo di Messer Bello Mancini illustrati da Robert Black (1996b: 731

5 Alle indicazioni minime su autore, denominazione, origine e periodo di composizione, facciamo seguire, entro parentesi tonde, una sigla e il principale riferimento bibliografico. Ulteriori segnalazioni in Thurot 1869: 54-56, 92. Da tenere presente anche il testo «inedito, probabilmente pavese, del primo Quattrocento» menzionato da Sabatini (1996: 431, con citazione di due themata): nella nota corrispondente (1996: 460 n. 14) lo studioso annuncia un suo futuro intervento sull'argomento («Ne darò notizia in altra occasione»).

6 Difficile pronunciarsi sul luogo di composizione di questi ultimi frammenti, oggi custoditi presso l'Archivio di Stato di Cuneo; il loro editore (Piccat 1988: 865) ricorda che «non contengono particolari indicazioni cronologiche o di composizione: i pochi toponimi riscontrabili [...] paiono restringere ad un'area lombardo-emiliana la diffusione del testo».

7 Copia di NdA, parziale e con talune divergenze rispetto all'originale, probabilmente allestita nella regione pugliese.

8 Interessante, alle pp. 36-37, il tentativo di legatura con altri esercizi di traduzione di area nordorientale sulla base di motivi e formule ricorrenti: «Si potrebbe dunque, avvicinando tutti questi testi, tracciare una linea culturale e geografica non spezzata, leggibile in un arco di tempo sincronicamente accettabile (dalla metà del sec. XII [sic] e forse da poco prima, a tutto il seguente, lungo la fascia Bergamo $[F B g$ e $R B g$ ], Verona $[G V r]$, Treviso [cf. Stussi 1968], Cividale $[E F]$, nella cui linea si inserirebbe [...] il nostro testo)» (citazione a p. 36). 
sgg.). ${ }^{9}$ In tal senso, la menzione degli esercizi di Cividale pubblicati da Schiaffini (1922) rappresenta un'eccezione solo parziale, dato il loro saldo legame con il frammento grammaticale di analoga provenienza. ${ }^{10}$

Un approfondimento, pur minimo, spetta senza dubbio a due opere fondamentali per lo sviluppo successivo della materia: le Regulae grammaticales di Francesco da Buti, composte a Pisa tra il 1355 e il 1378, e le omonime Regulae grammaticales di Guarino Guarini Veronese, redatte durante il soggiorno veneto (a Verona o più probabilmente a Venezia) ante 1426.

Le prime, che secondo Robert Black (1996b: 729) costituiscono «the most important intermediate Latin grammar of the fourteenth century», possono vantare una consistente tradizione manoscritta, studiata analiticamente da Fabrizio Franceschini (2003) in direzione geolinguistica: ${ }^{11}$ sua caratteristica distintiva è la diversa realizzazione su base regionale degli esempi volgari corrispondenti agli enunciati esemplificativi della grammatica latina, caratteristica che ha spinto lo studioso a costruire per i ventisette codici allora noti un piccolo atlante linguistico ante litteram di dieci lemmi della lingua comune (verbi atmosferici) osservati su tutto il territorio nazionale.

Le Regulae del celebre umanista Guarino Guarini' ${ }^{12}$, subito popolarissime e giunte, attraverso molteplici alterazioni e riduzioni, fino al secolo XVII, paiono comunque legate alla tradizione grammaticale medievale: il giudizio di Remigio Sabbadini (1896: 39, 45) risulta in tal senso assai netto, quando sostiene che «il metodo delle Regulae è tutto medievale» e che «tutto l'organismo [dell'opera] è medievale, senza il minimo indizio di quella ribellione, che ci hanno avvezzati a scorgere nell'indirizzo umanistico»; ${ }^{13}$ distante la posizione di W. Keith Perceval, il quale, partendo proprio da una rivisitazione del rapporto

9 Da parte nostra, possiamo aggiungere una raccolta inedita di esercizi grammaticali conservata oggi oltre i confini europei, su cui torneremo in altra sede.

10 Così il curatore a p. 95: «Che ci si facciano avanti, in un medesimo codice, il trattato teorico $[F F]$ e le applicazioni relative $[E F]$, è certo notevole». E una certa unitarietà di fondo deve probabilmente essere ravvisata anche per i diversi contributi grammaticali, tra cui alcuni themata, di cui si compone il frammento comense (FCo) pubblicato in Milani 2005.

11 Sull'importanza della variabile diatopica per gli scritti grammaticali latino-volgari, al di là dell'esempio estremo di Francesco da Buti, si legge a p. 64: «Nella tradizione di questi testi abbiamo insomma una forte diversificazione linguistica, connaturata con l'impiego del volgare in funzione della comprensione e memorizzazione di voci latine: ogni maestro o copista, per garantire a studenti e lettori un ausilio didattico sicuro, tende a riprodurre testi scolastici provenienti da altra area rispettando il latino ma sostituendo al volgare originario quello proprio o del proprio pubblico; allo stesso modo, se certi esempi grammaticali recano indicazioni toponomastiche, queste possono essere integrate o sostituite con nomi di luogo più familiari a scriventi e lettori potenziali» (poco oltre si parla di «processo di rideterminazione diatopica»). L'argomento è al centro del successivo Franceschini 2005.

12 Ad oggi prive di edizione moderna.

13 Diversamente, secondo Charles Thurot (1869: 491), nelle Elegantiae linguae latinae, vero e proprio «manifeste de la guerre que les humanistes déclaraient à la tradition scolastique en grammaire», Lorenzo Valla «rompt violemment avec le passé». 
con i manuali grammaticali precedenti, cerca piuttosto di dimostrare l'originalità delle posizioni guariniane. ${ }^{14}$

Due elementi accomunano i suddetti testi distinguendoli dagli altri segnalati: da una parte, la statura dei rispettivi autori, Francesco e Guarino, veri e propri teorici della materia grammaticale e non semplici maestri elementari (ludi magistri) come la maggior parte degli estensori, non di rado anonimi, delle altre opere indicate $;{ }^{15}$ dall'altra, ancor più rilevante, il notevole successo da essi riscontrato, un successo di portata sovra-regionale, comprovato dal numero certo non trascurabile dei testimoni manoscritti e delle edizioni antiche giunti fino a noi. ${ }^{16}$

Al contrario, gli altri trattati, conservati in codices unici salvo rare eccezioni, rappresentano tendenzialmente una realtà culturale e linguistica circoscritta: generalmente piccoli sussidiari per scuole locali e non importanti manuali ad ampia tiratura.

In questo senso, gli scritti di Francesco e di Guarino possono, almeno teoricamente, ricoprire il ruolo di intermediari, di connettori tra scritti geograficamente distanti; così avviene per esempio per i friulani $F F$ (e $E F$ ) e il salentino NdA (da cui anche NdAN), entrambi debitori del trattato pisano, almeno stando alle riflessioni dei rispettivi curatori: per $F F$, «E anche la Grammatica, a giudicar dai Frammenti, non è molto elevata: ha stretti rapporti, p. es., con quelle, tutt'altro che sublimi, composte dal Maestro italiano Cesare ${ }^{17}$ (sec. XIII) e da Francesco di Bartolo da Buti»; ${ }^{18}$ sull'altro versante, per NdA, «il trattato [...] prosegue riorganizzando materiali propri e fonti comuni alla tradizione grammaticale precedente, a partire dall'esponente forse più fortunato, le Regule grammaticales (13551378) del pisano Francesco da Buti» ${ }^{19}$, «in generale, non saremmo lontani dal vero affermando che coincidenze, a volte stringenti, con brani delle Regule attraversano tutto

14 Cf. in primo luogo Percival 1972; cf. anche Percival 1978.

15 In una sorta di posizione intermedia possiamo collocare Goro d'Arezzo, esponente del prestigioso studium aretino, Cristiano da Camerino, insegnante di grammatica e retorica a Perugia, definito da Coluccio Salutati «litterator sive grammaticus» (cf. Franceschini 2003: 64 n. 15), e Nicola de Aymo, cappellano della regina Maria d'Enghien forse insignito del titolo di magister theologiae (cf. Coluccia / Greco / Scarpino 2005: 117-121; per ulteriori rimandi bibliografici anche Coluccia 1990: 90 n. 50).

16 «Il successo e la diffusione della grammatica butiana potrebbero essere apprezzati esaminando sistematicamente gli antichi cataloghi o elenchi di libri»e, ancora, «nella fase precedente [all'avvento dei manuali umanistici] la grammatica del Buti gareggia con testi europei del calibro del Doctrinale o del Graecismus» (Franceschini 2003: 52, 54), ma un discorso simile vale anche per Guarino.

17 In realtà il cremonese Pietro da Isolella, autore di un compendio grammaticale pubblicato in Fierville 1886 (cf. supra).

18 Schiaffini 1922: 95-96.

19 Coluccia / Greco / Scarpino 2005: 122; alla corrispondente nota 2 si osserva che, sulla base della prossima edizione critica delle Regulae, "andrà stabilito se qualche elemento testuale di possibile collegamento tra le Regulae grammaticales e l'Interrogatorio [...] indichi un rapporto effettivo tra i due testi o se vada attribuito a fonti comuni o magari a mediazioni oggi impossibili da precisare». 
l'Interrogatorio $»^{20}$, e, ancora, «si evidenzia il ruolo preminente che le Regule assumono per la redazione dell'Interrogatorio». ${ }^{21}$

In questa sede vorremmo tuttavia richiamare l'attenzione su un altro intreccio testuale, che coinvolge almeno quattro scritti sopra segnalati.

Il punto di partenza, sorta di capostipite di una familia operum, si deve probabilmente identificare in GdA, il trattato composto da Maestro Goro assai probabilmente presso lo studium aretino nella prima metà del XIV secolo. ${ }^{22}$

Alcune tracce si rinvengono nello stesso Interrogatorio del leccese Nicola de Aymo $(\mathrm{NdA})$, visto che «Identica nei due autori è la classificazione di cinque generi dei nomi [...], mentre nelle altre grammatiche del XIV e XV sec., ad es. Buti e Guarino Veronese, vengono elencati sette generi».23

In altra direzione, un ramo della sua tradizione indiretta ci conduce a $F C n$, come detto da assegnare forse all'area lombardo-emiliana: «All'interno della tradizione grammaticale latina medievale il testo riportato [in $\mathrm{FCn}$ ] è in stretta dipendenza dalle Regulae parvae Magistri Gori de Aretio».. ${ }^{24}$

A comprovare tale ipotesi viene richiamata l'attenzione sul passo relativo al verbo neutro passivo, nella versione originale di Maestro Goro e in quella derivata di FCn, che qui vale la pena recuperare: ${ }^{25}$

Maestro Goro d'Arezzo, Regulae parvae (GdA):26 Nota quod verbum neutrum passivum est illud quod desinit in $o$ et non format passivum in or et vult ante se nominativum persone pacientis et post se ablativum, $a$ vel $a b$ mediante, persone agentis, ut vapulo a magistro: et sunt ista

$F C n$, cap. VII: ${ }^{27}$ Secundo. Notandum est quod verbum neutrum passivum est illud quod dessinit in $o$ et non format passivum in or et vult ante se nominativum persone pacientis et post se ablativum, $a$ vel $a b$ mediante, persone agentis, ut vapulo a magistro: et sunt ista

E tuttavia, sempre muovendoci tra le definizioni del verbo, in particolare del verbo neutro, lo stesso modello ci porta ad altro, ben più celebre discendente, le Regulae grammaticales di Guarino: ${ }^{28}$

20 Coluccia / Greco / Scarpino 2005: 132.

21 Coluccia / Greco / Scarpino 2005: 133; il puntuale confronto tra i due trattati, che, con ampie citazioni testuali, occupa le pp. 130-134, chiama in causa anche il testo grammaticale di Cristiano da Camerino (CdC).

22 Sull'autore si veda in primo luogo D'Alessandro 2006; della stessa studiosa segnaliamo anche la comunicazione D'Alessandro 2005. Cf. inoltre Black 1996b: 727-728.

23 Coluccia / Greco / Scarpino 2005: 130.

24 Piccat 1988: 865.

25 Nelle citazioni seguenti, al di là della veste grafica presente nella fonte, utilizziamo il corsivo per evidenziare singoli termini (solitamente voci grammaticali ed esempi dei fenomeni illustrati), riservando il maiuscoletto alle notazioni etimologiche.

26 Marchesi 1910: 51.

27 Piccat 1988: 865.

28 Entrambi i passi citati in Marchesi 1910: 41. 
Maestro Goro d'Arezzo, Regulae parvae (GdA): Nota quod verbum neutrum acquisitivum est illud quod desinit in $o$ et non format passivum in or, et vult ante se nominativum persone agentis et post se dativum persone pacientis, ut servio tibi

Guarino Guarini, Regulae grammaticales (GG): Nota quod verbum neutrum acquisitivum est illud quod vult ante se nominativum agentem et post se dativum patientem, ut ego servio tibi

Somiglianza ancor più marcata tra $\mathrm{i}$ due testi per la regola e gli esempi del verbo comune: ${ }^{29}$

Maestro Goro d'Arezzo, Regulae parvae (GdA): Nota quod verbum commune est illud quod habet utramque significationem .s. activam et passivam; quando habet activam vult ante se nominativum persone agentis et post se accusativum persone patientis, ut ego largior equum; sed quando habet passivam vult ante se nominativum persone patientis et post se ablativum $a$ vel $a b$ mediante, ut equus largitur a me. Et sunt ista: Largior per donare e per essere donato. Experior per sperimentare e per essere sperimentato. Veneror per honorare e per essere honorato. Moror per aspectare e per essere aspectato. Osculor per basciare e per essere basciato. ${ }^{*}$ Criminor per incolpare e per essere incolpato. ${ }^{\circ}$ Amplector per abraciare e per essere $^{30}$ abraciato. ${ }^{\bullet}$ Hospitor per albergare e per essere albergato. ${ }^{\square}$ Hortor per confortare e per essere confortato. Largior experior veneror moror osculor ortor, / Criminor amplector tibi sunt communia $^{31}$, lector. / Si bene connumeres interpretor hospitor addere debes. Amen.

Guarino Guarini, Regulae grammaticales (GG): Nota quod verbum commune est illud quod desinit in or et non formatur a verbo desinente in $o$. Et duplicem habet significationem, scilicet activam et passivam. Quando habet activam vult ante se nominativum agentem et post se accusativum patientem, ut ego largior tibi librum. Quando habet passivam vult ante se nominativum patientem et post se ablativum agentem cum $a$ vel $a b$, ut liber largitur a me tibi. Largior per donare et esser donato. Experior per isperimentare et esser isperimentato. Veneror per honorar et esser honorato. Moror per ritardar et esser ritardato. Osculor per basciar et esser basciato. ${ }^{\square}$ Hortor per confortare et esser confortato. ${ }^{*}$ Criminor per incolpare et esser incolpato. ${ }^{\circ}$ Amplector per abbracciare et esser abbracciato. Interpretor per esporre et esser esposto. -Hospitor per albergare et essere albergato. Largior experior veneror moror osculor hortor, / Criminor amplector tibi sunt communia, lector. / Si bene connumeres interpretor hospitor addes.

Particolare rilievo congiuntivo per l'ultimo verso citato, ricavato, come i due precedenti, dal Doctrinale di Alexandre de Villadieu ${ }^{32}$, ove si legge «Et bene si numeres interpretor

29 Marchesi 1910: 41-42; evidenziamo con richiami grafici i casi di diverso ordinamento della materia; inseriamo il tratto obliquo a separare le tre frasi finali («Largior [...] ortor», «Criminor [...] lector», «Si bene [...] debes» nella forma di $\mathrm{GdA})$, corrispondenti a tre versi tratti dal Doctrinale (cf. infra).

30 In Marchesi 1910: 42 erroneamente trascritto assere, corretto a p. 56.

31 La virgola seguente, assente in Marchesi 1910: 42, è stata ricavata dal testo di p. 56 e da questo estesa al corrispondente passo di GG.

32 Edizione moderna: Reichling 1893 
addere debes»: evidentemente Goro inserisce hospitor e, attraverso questo passaggio, Guarino restituisce la corretta misura collocando addes per addere debes..$^{33}$

Se è vero che il problema dell'identificazione delle fonti utilizzate da Guarino, esplorato nei dettagli nei già menzionati studi di W. Keith Percival $(1972$; 1978), resta di difficile soluzione, simili consonanze sembrano poter avvalorare un rapporto diretto con il modello di Goro, come già ipotizzato da Concetto Marchesi (1910: 40-41): «E veramente queste regole di Goro presentano grande somiglianza con le famose regulae guariniane [...]. Alcuna volta [...] la regula guariniana è una fedele ripetizione del dettato di Goro, nelle espressioni e negli esempi».

Seguendo questa concatenazione di riprese testuali, si può introdurre un nuovo anello strettamente legato all'esempio guariniano, le Regule composte a Venezia nel 1432 da Maffeo Valeresso (MV): «Questi, per l'età sua, non frequentò probabilmente la scuola veneziana di Guarino, ma ne risentì certo l'influsso. Per la sua compilazione adotta il titolo dell'opera guariniana e la tiene sempre dinanzi: inverte l'ordine delle regole, ne amplia od abbrevia qualcuna, si vale più volte delle parole stesse e degli esempi del suo modello». ${ }^{34}$

Ma il trattato di Maffeo risente certo di sollecitazioni molteplici, alcune da assegnare forse a Francesco da Buti ${ }^{35}$, altre, eventualmente giunte attraverso il tramite dello stesso Francesco, da ricondurre alla precedente tradizione latina.

Tra queste ultime, probabilmente, l'enumerazione delle otto parti del discorso, che così leggiamo in Maffeo: ${ }^{36}$

MV: Partes orationis sunt octo, scilicet: nomen, verbum, participium, pronomen, praepositio, adverbium, interiectio et coniunctio.

Il medesimo ordine si rinviene anche in Guarino («nome verbo participio pronome; preposiz. avverbio interiezione congiunz») $)^{37}$ e prima di lui, come rilevato da Percival (1972: 266), «in a great many grammars of the Middle Ages, for instance in the grammar of Pietro da Isolella from the thirteenth century, and in both fourteenth century grammars from which Sabbadini himself quotes, i.e., the grammars of Folchino dei Borfoni and Francesco da Buti».

Citiamo proprio da quest'ultimo: ${ }^{38}$

FdB: Octo, scilicet nomen, verbum, participium, pronomen, prepositio, adverbium, interiectio et coniunctio.

Analogamente, nelle Regulae parvae di Maestro Goro: ${ }^{39}$

33 Cf. Marchesi 1910: 42 n. 1; nella citazione di p. 42 addere è posto tra parentesi quadre, assenti per il testo riportato a p. 56.

34 Segarizzi 1915-1916: 90.

35 Segarizzi 1915-1916: 91 n. 1.

36 Segarizzi 1915-1916: 91.

37 Sabbadini 1896: 43.

38 Franceschini 2003: 63.

39 Marchesi 1910: 45. 
GdA: Quot sunt partes orationis? Sunto octo: que? nomen verbum participium pronomen prepositio adverbium interiectio et coniunctio.

E si aggiunga ancora $F C o$, che nelle sezioni che conservano l'originario trattato lascia intravedere la successione verbo, participio, pronome, e che, soprattutto, nelle successive definizioni delle parti del discorso presenta la sequenza completa nome, verbo, participio, pronome, preposizione, avverbio, interiezione e congiunzione. ${ }^{40}$

Riscontro parziale, ma non trascurabile anche per i $F F$, la cui trattazione, forse attraverso l'esempio di Francesco da Buti, segue l'ordine nome, verbo e participio.

Teoricamente, recuperando anche alcune indicazioni di parentela rilevate in precedenza, si potrebbe ipotizzare una contaminazione testuale interna alla tradizione delle grammatiche latino-volgari. Tuttavia, la precocità ${ }^{41}$, l'ampiezza e l'articolazione delle testimonianze offerte lasciano piuttosto pensare alla conservazione e alla riproposizione, con riprese dirette o mediate, di un comune antecedente latino.

E questo antecedente deve essere identificato in ultima analisi con Prisciano e le sue Institutiones ${ }^{42}$, dove la trattazione delle parti del discorso segue il medesimo ordine nome (libri II-VII), verbo (VIII-X), participio (XI), pronome (XII-XIII), preposizione (XIV), avverbio e interiezione (XV).

Tuttavia, tra questo autorevole modello e le realizzazioni tardo-medievali esiste almeno un altro manuale di larghissima diffusione che nominalmente chiama in causa l'altro grande grammatico della latinità, Elio Donato: ${ }^{43}$ il Donatus o Donatellus, oggi meglio noto con il titolo di Ianua. ${ }^{44}$

Alcune rapide precisazioni sul contenuto e sulla genesi dell'opera: ${ }^{45}$ nonostante l'attribuzione a Donato e la forma dialogica comune con quella dell'Ars minor, il trattato recupera in realtà l'impostazione e le definizioni delle Institutiones di Prisciano. Come ben rilevato da Robert Black (1996a: 11), tale scelta si può collocare all'interno di una progressiva «dissatisfaction [...] with Donatus's Ars minor», un testo che, ben lontano dal coprire tutte le sezioni della materia grammaticale e per di più privo di esempi completi di declinazioni nominali e di paradigmi verbali, costituiva uno strumento didattico inadeguato per la piena età medievale, quando il latino aveva ormai perso il ruolo di lingua prima. ${ }^{46}$

40 Milani 2005: 333-336.

41 Soprattutto con l'attestazione di Pietro da Isolella.

42 Edizione moderna: Hertz 1855.

43 Celebre autore dell'Ars minor e dell'Ars maior, entrambe edite in Holtz 1981.

44 Titolo ricavato da Remigio Sabbadini (1896: 43) dalla prima parola del prologo che solitamente precede il trattato grammaticale: «Ho dunque ragione io di credere che il manuale del corso elementare guariniano non fosse il Donatus minor, ma la Ianua, che ne è del resto una derivazione medievale. La denomino così, perché essa porta in capo quattro distici, di cui il primo verso suona: 〈Ianua sum rudibus primam cupientibus artem»». Edizioni moderne: Bologna 1980; Schmitt 1969.

45 Sintetica descrizione offerta da Robert Black (1996b: 703-704): «Donatus or Donadello, a treatise on Latin morpgology and accidence, divided into eight sections corresponding to the eight parts of speech, now known to scholars as Ianua».

46 Percival 1988: 69: «Ars minor of Donatus [...] served as a beginner's manual - it provided nothing more than a definitional framework conveniently formulated in question-and-answer format for use in the classroom». 
Il nuovo quadro linguistico sollecitava dunque l'inserimento di alcune integrazioni al dettato originale di Donato e, in un secondo tempo, l'approntamento di veri e propri rimaneggiamenti, simili al modello latino nella struttura dialogica, profondamente rivisitati nei contenuti.

Seguendo ancora il pensiero di Robert Black (1996a: 11), i grammatici medievali cominciarono a produrre essi stessi i loro trattati di grammatica elementare per sostituire l'Ars minor. ${ }^{47}$ In questa operazione cominciò a farsi strada l'impostazione analitica ${ }^{48}$, favorita dalla suddivisione della stessa Ars minor nelle otto sezioni dedicate alle parti del discorso e dalla sua forma dialogica; tuttavia, alle domande di carattere generale che caratterizzano l'Ars minor subentrano quesiti particolari sulle singole parole scelte per esemplificare ciascuna categoria grammaticale, mentre le riflessioni teoriche complessive sono introdotte a chiarimento di esempi specifici. Questo nuovo tipo di grammatica non esclude l'impiego di definizioni e di terminologia tratte da Donato, ma la fonte primaria tende progressivamente a identificarsi con le Institutiones di Prisciano.

Durante questo processo, intorno al XII-XIII secolo si andarono costituendo in Europa due diverse tradizioni testuali e, parallelamente, pedagogiche: nei paesi centro-settentrionali trovò larga diffusione la grammatica analitica nota come Remigius ${ }^{49}$, che utilizza come nome esemplificativo «Dominus», mentre in Italia prevalse decisamente il già menzionato rimaneggiamento Ianua, il cui sostantivo esemplificativo è «Poeta»: «manuscripts of parsing grammars with the incipit 〈Dominus que pars est〉 [Remigius] are almost entirely northern European, with the majority from Germany [...]. On the other hand, Ianua manuscripts are primarily Italian». ${ }^{50}$

Tornando sui nostri passi, basti sottolineare che i due testi si distinguono anche per l'ordine seguito nella trattazione delle otto parti del discorso, per le quali lo Ianua presenta la stessa sequenza di Prisciano, mentre il Remigius conserva quella di Donato: ${ }^{51}$

Prisciano - Ianua: Nomen («Poeta»), Verbum, Participium, Pronomen, Praepositio, Adverbium, Interiectio, Coniunctio

Donato - Remigius: Nomen («Dominus»), Pronomen, Verbum, Adverbium, Participium, Coniunctio, Praepositio, Interiectio

47 Opera che pure continua a essere tradotta fino al pieno Quattrocento: per le molteplici e variamente elaborate versioni di ambito francese si veda la trattazione complessiva di Maria Colombo Timelli (1996).

48 Sull'esempio delle Partitiones di Prisciano, nelle quali viene minuziosamente analizzato il primo verso di ciascun libro dell'Eneide.

49 Edizione moderna: Pinborg 1982; così il curatore a p. 65: «The so-called Remigius is a representative of a huge family of treatises dealing with latin morphology or accidence, written in the popular form of question an answer - exactly as the Donatus minor. The name Remigius alludes to the famous Carolingian grammarian who wrote commentaries on Donatus, but the treatise as we know it has probably no direct relation to Remigius».

50 Black 1996a: 12.

51 La seguente tavola è ripresa, con qualche lieve modifica, da Pinborg 1982: 65-66. 
Pur rilevata la piena corrispondenza tra la sequenza presente nello Ianua e quella che caratterizza le grammatiche latino-volgari menzionate ${ }^{52}$, per alcuni testi resta assai arduo dimostrare un'eventuale discendenza diretta: così, nel commento al trattato guariniano, mentre Sabbadini (1896: 42-43) utilizza proprio l'argomento dell'ordine delle parti del discorso come una delle prove della dipendenza dallo Iаnиa, Percival (1972: 266) rileva che «The fact that this order is found in both the Ianua and Guarino's Regulae does not prove that the two works have any special historical connexion with each other» ${ }^{53}$

In casi simili la questione è forse destinata a rimanere insoluta, almeno in assenza di studi critici esaustivi su tutti i testi coinvolti. ${ }^{54}$

In altri il ruolo di intermediazione -sorta di scritto interpositus- svolto dallo Ianua tra Prisciano e l'esito tardo-medievale pare essere difficilmente contestabile, come per le definizioni contenute in $\mathrm{FCo}::^{55}$

Priscianus, Institutiones, II, 22:56 Nomen est pars orationis, quae unicuique subiectorum corporum seu rerum communem vel propriam qualitatem distribuit. Dicitur autem nomen vel a Graeco, quod est NOMA et adiecta O ONOMA, dictum a tribuendo, quod NEMEIN dicunt, vel, ut alii, nomen quasi notamen, quod hoc notamus uniuscuiusque substantiae qualitatem. Et communem quidem corporum qualitatem demonstrat, ut homo, propriam vero, ut Virgilius, rerum autem communem, ut disciplina, ars, propriam, ut arithmetica Nicomachi, grammatica Aristarchi.

Ianua, Firenze, Biblioteca Laurenziana, Ms. Laurenziano Strozzi 80, f. 1r: ${ }^{57}$ Quid est nomen? Nomen est pars orationis declinabilis que unicuique subiectorum corporum seu rerum propriam vel communem qualitatem distribuit, communem quidem corporum ut homo, propriam ut Virgilius. Unde dicitur nomen? Nomen dicitur a NOMA greco vel a NOTAMINI, eo quod per ipsum notemus qualitatem uniuscuiusque substantie. Quid est proprium nominis? Proprium nominis est significare substantiam, qualitatem cum casu.

FCo, Definizioni: ${ }^{58}$ Quid est nomen? Nomen est pars orationis declinabilis que unnicuique subditorum corporum seu rerum comunem vel propriam qualitatem distribuit: comunem qualitatem corporum ut homo, propriam ut Vergilius; comunem quidam rerum ut ars et

52 Situazione particolare per l'Interrogatorio di Nicola de Aymo (NdA): nel Trattato I, dedicato all'enumerazione delle parti del discorso, si rispetta l'ordine tipico di Donato nome, pronome, verbo, avverbio, participio, congiunzione, preposizione, interiezione; diversamente, la trattazione successiva offre la sequenza di Prisciano nome, verbo, participio, pronome, preposizione, avverbio, interiezione e congiunzione (cf. Greco 2008).

53 Poco oltre, a p. 268, lo studioso precisa quanto segue: «In conclusion, it seems to me that Sabbadini failed to demonstrate that a historical connexion existed between the Ianua and Guarino's Regulae. [...] It should be also clear, however, that I have not proved the contrary, i.e., that Guarino was not influenced by the Ianua».

54 Ancora Percival (1972: 268) ammette per Guarino che «When we observe a Priscianic formulation being shared by a number of mediaeval grammars and by Guarino's Regulae, it is not possible to establish the chain of transmission from those facts alone».

55 Chiuse tra l'altro dall'explicit «Finis. Donateli hic est» (c. 27r).

56 Hertz 1855: 1, 56-57.

57 Black 1992: 103.

58 Milani 2005: 313. 
disciplina, propriam ut Grammatica Prisciani et Arismetica Nicomaci. Quid est proprium nominis? Propium nominis est significare substantiam et qualitatem propriam vel comunem cum casu. Unde dicitur nomen? Nomen a NOMA greco vel a NOTAMINI, // eo quod per ipsum substantiam et qualitatem unicuiusque rei notemus.

Partiti da Goro d'Arezzo (GdA), dopo aver toccato, in direzioni opposte, lo scritto leccese di Nicola de Aymo (NdA) e un trattato di origine lombardo-veneta $(F C n)$, siamo passati in ambiente veneto con Guarino, giungendo, per suo tramite, a Maffeo (MV); di qui siamo risaliti ancora a Guarino e a Goro, allargando il nostro percorso fino a comprendere Francesco da Buti, i frammenti friulani $(F F)$ e quello comense $(F C o)$, senza dimenticare i testi di Pietro da Isolella e di Folchino dei Borfoni, per ipotizzare infine, alle loro spalle, un'influenza di Prisciano, probabilmente attraverso la mediazione dello Ianua.

Un cammino certo tortuoso e ancora ricco di punti oscuri, che tuttavia presenta forse il merito di collegare testi variamente disseminati nel tempo e nello spazio della nostra penisola.

\section{Bibliografia}

Baldelli, Ignazio (1959): L'edizione dei glossari latino-volgari dal secolo XIII al XV. In: Atti dell'VIII Congresso internazionale di studi romanzi. Firenze 3-8 aprile 1956. Vol. 2. Firenze: Sansoni, $757-$ 763.

Belloni, Gino / Pozza, Marco (1987): Sei testi veneti antichi. Roma: Jouvence.

Black, Robert (1992): An unknown thirteenth-century manuscript of Ianua. In: Wood, Ian / Loud, G.A. (edd.): Church and Chronicle in the Middle Ages. Essays Presentend to John Taylor. London: Hambledon press, 101-115.

- (1996a): Ianua and elementary education in Italy and Northern Europe in the later middle ages. In: Tavoni, Mirko (ed.): Italia ed Europa nella linguistica del Rinascimento: confronti e relazioni. Atti del Convegno internazionale, Ferrara 20-24 marzo 1991. Vol. 2. Modena: Panini, 5-22.

- (1996b): The vernacular and the teaching of latin in thirteenth and fourteenth-century Italy. In: StM 37, 2, 703-751.

Bocchi, Andrea (2005): L'edizione di un glossario latino-volgare ad attestazione plurima. In: Filologia Italiana 2, 105-135.

Bologna, Giulia (ed.) (1980): Libri per una educazione rinascimentale. Grammatica del Donato Liber Iesus (1 vol. + 2 ripr.). Milano: Comune di Milano.

Casapullo, Rosa (1999): Il Medioevo. Bologna: Il Mulino.

Colombo Timelli, Maria (1996): Traductions françaises de l'Ars minor de Donat au Moyen Age (XIII $-X V^{e}$ siècles). Firenze: La Nuova Italia.

Coluccia, Rosario (1990): Notai pugliesi, grafie e storia linguistica. In: SLI 16, 80-96.

- / Greco, Rosa Anna / Scarpino, Cristina (2005): L'Interrogatorio di Nicola de Aymo: una grammatica latino-volgare leccese del 1444. In: Medioevo Letterario d'Italia 2, 117-144.

Contini, Gianfranco (1934): Reliquie volgari dalla scuola bergamasca dell'umanesimo. In: ID 10, 223-240.

D’Agostino, Alfonso (1983): Antichi glossari latino-bergamaschi. In: Studi di lingua e letteratura lombarda offerti a Maurizio Vitale. Vol. 1. Pisa: Giardini Editori e Stampatori, 79-111. 
D'Alessandro, Teresa (2005): I manoscritti di Goro d'Arezzo. Comunicazione tenuta in occasione della «Giornata di studi in memoria di I Deug-Su», Dipartimento di Teoria e Documentazione delle Tradizioni Culturali, Arezzo 24 gennaio 2005.

- (2006): Goro d'Arezzo maestro di grammatica e commentatore di Lucano. In: Stella, Francesco (ed.): 750 anni degli statuti universitari aretini. Atti del convegno internazionale su origini, maestri, discipline e ruolo culturale dello Studium di Arezzo, Arezzo 16-18 febbraio 2005. Firenze: SISMEL - Edizioni del Galluzzo.

De Santis, Carla (ed.) (2003): Folchini de Borfonibus Opera Omnia. Pars I, Cremonina (gramatica, orthographia et prosodia). Turnhout: Brepols.

De Stefano, Antonino (1905): Una nuova grammatica latino-italiana del secolo XIII. In: RLaR 48, 495-529.

Fierville, Charles (1886): Une grammaire latine inédite du XIII siècle. Paris: Imprimerie Nationale.

Franceschini, Fabrizio (2003): Le Regule di Francesco da Buti tra scuola laica e Osservanza: un atlante linguistico dell'Italia trecentesca. In: CoFIM 17, 51-130.

- (2005): Variation linguistique et toponomastique comme marque d'identité dans les grammaires du Moyen Age et de la Renaissance. In: Chiorboli, Jean (ed.): Parcours Interculturels. Circulation des idèes, des hommes, des livres et des cultures. Biguglia: Sammarcelli, 231-245.

Gasca Queirazza, Giuliano (1966): Documenti di antico volgare in Piemonte. Fasc. 3: Frammenti vari da una Miscellanea Grammaticale di Biella. Torino: Bottega d'Erasmo.

Greco, Rosa Anna (2008): La grammatica latino-volgare di Nicola de Aymo (Lecce, 1444): un dono per Maria d'Enghien. Galatina: Congedo.

Gualdo, Riccardo (1999): L'uso dei glossari latino-volgari in area lombardo-veneta nel primo Quattrocento. In: Gasparino Barzizza e la rinascita degli studi classici fra continuità e rinnovamento. Atti del Seminario di studi, Napoli 11 aprile 1997. Napoli: Istituto Universitario Orientale, 209-246.

Hertz, Martin (ed.) (1855): Prisciani grammatici Caesariensis institutionum grammaticarum libri 18 (2 voll.). Lipsiae: Teubner.

Holtz, Louis (1981): Donat et la tradition de l'enseignement grammatical. Etude sur l'Ars Donati et sa diffusion (IV $V^{e}-I X^{e}$ siècle) et édition critique. Paris: Centre National de la Recherche.

Lovito, A. (1994-1995): Appunti filologici e linguistici sul ms. V.C.4 della Nazionale di Napoli. Edizione critica, analisi linguistica, indici lessicali. Tesi di Laurea. Relatore Paolo Trovato. Salerno: Facoltà di Lettere e Filosofia dell'Università degli Studi di Salerno.

Manacorda, Giuseppe (1913-1914): Un testo di grammatica latino-veneta del sec. XIII. In: Atti della R. Accademia delle Scienze di Torino 49, 689-698.

Marchesi, Concetto (1910): Due grammatici latini del Medio Evo. In: Bullettino della Società Filologica Romana 12, 19-56.

Martinelli, Chiara (in corso di redazione): Edizione critica delle Regulae grammaticales di Francesco da Buti. Tesi di Dottorato di ricerca in Studi italianistici dell'Università di Pisa, tutor Grabriella Albanese e Fabrizio Franceschini.

Milani, Matteo (2005): "Al è più bello de tuti»: esercizi in volgare in un frammento grammaticale comense. In: La Parola del Testo 9, 2, 307-356.

Nardi, Bruno (1971): Saggi sulla cultura veneta del Quattro e Cinquecento. Padova: Antenore.

Percival, W. Keith (1972): The historical sources of Guarino's Regulae grammaticales. In Tarugi, Giovannangiola (ed.): Civiltà dell'Umanesimo. Atti del 6, 7, 8 Convegno internazionale del Centro di studi umanistici, Montepulciano 1969, 1970, 1971. Firenze: Olschki, 263-284.

- (1978): Textual problems in the Latin grammar of Guarino Veronese. In: Res publica litterarum 1, 241-254.

- (1988): Renaissance grammar. In: Rabil, Albert Jr.: Renaissance Humanism: Foundations, Forms and Legacy. Vol. 3: Humanism and the disciplines. Philadelphia: University of Pennsylvania press, 67-83. 
Piccat, Marco (1988): I frammenti grammaticali latino-volgari dell'Archivio di Stato di Cuneo. In: Miscellanea di Studi Romanzi offerta a Giuliano Gasca Queirazza. Vol. 2. Alessandria: Edizioni dell'Orso, 863-886.

Pinborg, Jan (ed.) (1982): Remigius, Schleswig 1486. A Latin Grammar in Facsimile Edition. København: DetKongelige Danske Videnskabernes Selskab.

Reichling, Dietrich (ed.) (1893): Alexander de Villa Dei, Doctrinale. Berlin: Hoffman \& Comp.

Rizzo, Silvia (1996): L'insegnamento del latino nelle scuole umanistiche. In: Tavoni, Mirko (ed.): Italia ed Europa nella linguistica del Rinascimento: confronti e relazioni. Atti del Convegno internazionale, Ferrara 20-24 marzo 1991. Vol. 1. Modena: Panini, 3-29.

Sabatini, Francesco (1963-1964): Il Glossario di Monza. In: Atti della R. Accademia delle Scienze di Torino 90, 51-84.

- (1996): Prospettive sul parlato nella storia linguistica italiana (con una lettura dell'Epistola napoletana del Boccaccio). In: Coletti, Vittorio / Coluccia, Rosario / D’Achille, Paolo / De Blasi, Nicola / Petrucci, Livio (edd.): Italia linguistica delle Origini. Saggi editi dal 1956 al 1996. Vol. 2. Lecce: Argo, 425-466.

Sabbadini, Remigio (1896): La scuola e gli studi di Guarino Guarini Veronese. Catania: Tip. F. Galati.

- (1904): Frammento di grammatica latino-bergamasca. In: StM 1, 281-292.

Schiaffini, Alfredo (1921): Frammenti grammaticali latino-friulani del secolo XIV. In: Rivista della Società Filologica Friulana 2, 1-2, 3-16 e 93-105. Pubblicato anche in estratto Udine: Tip. D. Del Bianco e Figlio.

- (1922): Esercizi di versione dal volgare friulano in latino nel secolo XIV in una scuola notarile cividalese. In: Rivista della Società Filologica Friulana 3, 2, 87-117. Pubblicato anche in estratto Udine: Società Filologica Friulana.

Schmitt, Wolfgang O. (1969): Die Ianua (Donatus). Ein Beitrag zur lateinischen Schulgrammatik des Mittelalters und der Renaissance. In: Beiträge zur Inkunabelkunde 4, 43-80.

Segarizzi, Arnaldo (1915-1916): Una grammatica latina del secolo XV. In: Atti del R. Istituto Veneto di Scienze, Lettere ed Arti 75, 89-96.

Stussi, Alfredo (1968): Esercizi di traduzione trevigiani del secolo XIV. In: ID 31, 23-29.

Thurot, Charles (1869): Extraits de divers manuscrits latins pour servir à l'histoire des doctrines grammaticales au Moyen Age. Paris: Imprimerie Impériale.

Trovato, Paolo (1998): Circolazione di libri e di varietà linguistiche nei conventi napoletani e abruzzesi. In margine alle Scritture volgari del Miòla. In: Trovato, Paolo: L'ordine dei tipografi. Lettori, stampatori, correttori tra Quattro e Cinquecento. Roma: Bulzoni, 91-127. Già comparso In: Trovato, Paolo (ed.) (1993): Lingue e culture dell'Italia meridionale, 1200-1600. Con una bibliografia delle edizioni di testi meridionali antichi, 1860-1914 a cura di Lidia Maria Gonelli. Roma: Bonacci, 269-307. 